\title{
Introduction to the Special issue on IEEE-Latin American Symposium on Circuits and Systems
}

\author{
Maria Teresa Sanz-Pascual • Arturo Sarmiento-Reyes • \\ Malgorzata Chrzanowska-Jeske
}

Published online: 15 August 2013

(C) Springer Science+Business Media New York 2013

This special issue of the Springer Analog Integrated Circuits and Signal Processing (ALOG) presents the selection of best papers from the Third IEEE Latin American Symposium on Circuits and Systems (LASCAS 2012). The symposium, a forum for discussion of the latest technical novelties on circuits and systems topics, was held in Playa del Carmen, Mexico, from February 29 to March 2, 2012.

112 papers from 20 countries and 3 continents were submitted to the conference. 69 papers were accepted based on the reviews from international experts. These contributions made up a high-quality technical program covering a wide variety of subjects in the areas of analog and digital circuits and systems, computer aided design, modeling and simulation, signal processing, sensor systems, high-frequency systems, voltage references, processor architectures and control theory.

From the papers presented at the conference, 12 papers were pre-selected for journal publication based on the evaluations of the Program Committee members and the corresponding session chairs. After a thorough revision of the extended papers, 7 papers were finally accepted for

M. T. Sanz-Pascual ( $\bigotimes)$ · A. Sarmiento-Reyes

Electronics Department, Instituto Nacional de Astrofísica,

Óptica y Electrónica, Luis Enrique Erro No. 1, Santa Maria

Tonantzintla, 72840 Puebla, Mexico

e-mail: materesa@inaoep.mx

A. Sarmiento-Reyes

e-mail: jarocho@inaoep.mx

M. Chrzanowska-Jeske

Electrical and Computer Engineering Department, Maseeh

College of Engineering and Computer Science, Portland State

University, 1900 SW Fourth Avenue, Portland, OR 97207-0751,

USA

e-mail: jeske@ece.pdx.edu publication in this special issue. These contributions are: (1) an ultra low power consumption millimeter-wave voltage controlled oscillator in a $65 \mathrm{~nm}$ CMOS-SOI technology; (2) a rail-to-rail differential quasi-digital converter for low-power applications; (3) analog sigma-delta modulation with op-amp gain compensation for nanometer technologies; (4) design of an integrated single-input dualoutput 3 -switch buck converter based on sliding mode control; (5) offset and gain calibration circuit for MIMISFET devices; (6) parallel algorithm for evolvable-based boolean synthesis on GPUs; (7) neural inverse optimal control applied to type 1 diabetes mellitus patients.

We thank the researchers from around the world who have chosen LASCAS 2012 for presenting their recent developments. We are deeply thankful to the reviewers for their contribution to the creation of the LASCAS 2012 technical program and for their involvement in the revision of the selected papers for this special issue. Finally, we thank the session chairs who not only contributed to the successful development of the symposium, but also helped in the selection of the best papers for journal publication. 


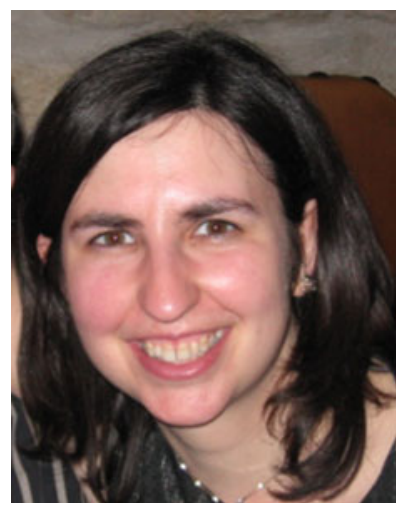

Maria Teresa Sanz-Pascual received the $\mathrm{Ph} . \mathrm{D}$. degree in Electronic Engineering from the University of Zaragoza, Spain, in 2004. She was a member of the Electronic Design Group at the Department of Electronic Engineering and Communications at the University of Zaragoza until 2008 and is currently a Full Researcher at the Electronics Department of the National Institute for Astrophysics, Optics and Electronics (INAOE), Mexico. Her research interests include analog and mixed IC design, integrated optical receivers and integrated sensor interfaces.

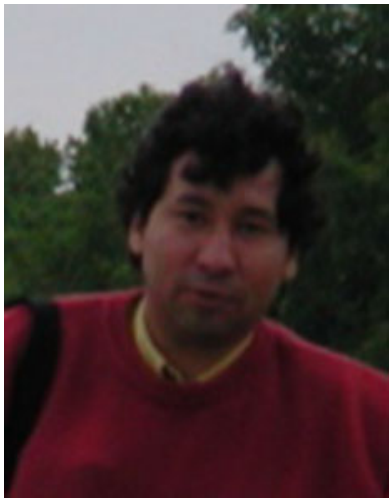

Arturo Sarmiento-Reyes Arturo Sarmiento-Reyes received the $\mathrm{Ph} . \mathrm{D}$. degree in Electronic Engineering from the Delft University of Technology in 1994. $\mathrm{He}$ was assistant researcher within the Electronics Laboratory at the TUDelft from 1989 to 1995. Since 1996 he is a Full Researcher at the Electronics Department of the National Institute for Astrophysics, Optics and Electronics (INAOE), Mexico, and currently he serves as Head of the Electronics Department. His topics of interest are design automation, nanoelectronic device modeling, and nonlinear circuits.

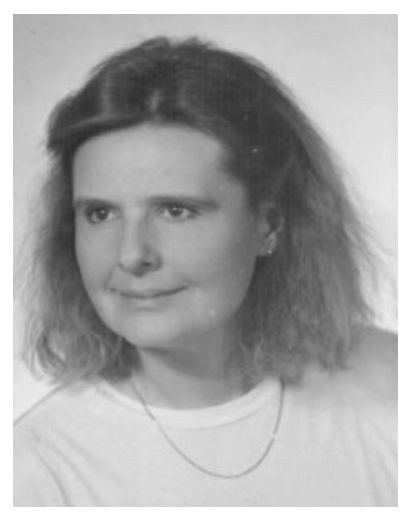

Malgorzata ChrzanowskaJeske Malgorzata ChrzanowskaJeske received her M.S. degree in electronics engineering from Politechnika Warszawska (the Technical University of Warsaw) in Warsaw, Poland, and the Ph.D. degree in electrical engineering from Auburn University, Auburn, Alabama. She has served on the faculty of the Technical University of Warsaw, and as a design automation specialist at the Research and Production Center of Semiconductor Devices in Warsaw.

Since 1989, she is with the Department of Electrical and Computer Engineering at Portland State University in Portland, Oregon, currently as a Professor. She was Chair of the ECE department for two terms from 2004 to 2010. Her research interests include CAD for VLSI IC, MS-SOC, 3D ICs, nanotechnology and nano/bio systems, design for manufacturability and design issues in emerging and renewable technologies. She has published more than 100 technical papers and serves as a panelist and a reviewer for the National Science Foundation (NSF), and as a reviewer for National Research Council Canada (NRC) and many international journals and conferences. Her research has been supported by the NSF and industry. Dr. Chrzanowska-Jeske has served on the Technical, Steering, and Organizing Committees of many international conferences, and was a Technical Chair of the 2002 International Conference on Electronics, Circuits and Systems and the 2012 Latin America Symposium on Circuits and Systems. In 2004, she was a Guest Editor of the International Journal on Analog Integrated Circuits and Signal Processing. She is serving her second term on the Board of Governors of IEEE Circuits and Systems Society (CASS) where she was also the Chair of the DLP program and the Chair of Women in CAS. She received the Best Paper Award from Alabama Section of IEEE for the best IEEE Transaction paper in 1990 and IEEE Council on Electronic Design Automation 2008 Donald O. Pederson Best Paper Award in IEEE Transactions on Computer-Aided-Design of Integrated Circuits and Systems. 